\title{
ICS as a Limiting Factor for Electron Energies in Pulsar Magnetospheres
}

\author{
Rodrigo Supper \\ Max-Planck-Institut für extraterrestrische Physik, Postfach 1603, 85740 \\ Garching, Germany
}

\begin{abstract}
Based on the polar cap model, we investigated in the energy loss of accelerated electrons in a neutron star magnetosphere by inverse Compton scattering of thermal photospheric radiation. An analytical treatment is presented, which turns out ranges in the magnetic field strength and thermal temperature where ultrarelativistic electrons can not survive.
\end{abstract}

\section{Theory of relativistic inverse Compton scattering}

Two kinds of inverse Compton scatter processes have to be considered: the nonresonant ('normal') and the resonant case. Additionally, beside the Thomson case the Klein-Nishina case has to be considered because of the ultrarelativistic energies of the electrons in the neutron star magnetosphere.

According to Feenberg \& Primakoff (1948) we can write for the mean energy transfer within a single inverse Compton collision in the non-resonant case (absence of an external magnetic field):

$$
\begin{aligned}
& \frac{d E^{I C S}}{d r} \cong-\sigma_{T} \int_{0}^{4 \pi}(1-\beta \cos \theta) d \Omega \int_{0}^{\epsilon(\theta)} n(\epsilon, \theta) \Delta E^{(1)} d \epsilon \\
& \quad-\frac{3}{4} \sigma_{T} \int_{0}^{4 \pi}(1-\beta \cos \theta) d \Omega \times \int_{\epsilon(\theta)}^{\infty} n(\epsilon, \theta) \frac{m_{e} c^{2}}{2 \epsilon^{*}} \ln \left(1+\frac{2 \epsilon^{*}}{m_{e} c^{2}}\right) \Delta E^{(2)} d \epsilon,
\end{aligned}
$$

with $\sigma_{T}=8 \pi / 3\left(e^{2} / m_{e} c^{2}\right)^{2}$ the Thomson cross section, $\epsilon$ the energy of the infalling photons in the laboratory frame, $\epsilon^{*}=\gamma(1-\beta \cos \theta) \epsilon$ the energy in their rest frame, $\theta$ the angle between the moving direction of the electron and the direction of the infalling photon stream, $n(\epsilon, \theta)$ the number of infalling photons per unit volume $d V$, energy range $d \epsilon$, and solid angle element $d \Omega$, and $m_{e}$ the rest mass of the electron. The first line on the right side of equation (1) gives the energy transfer in the Thomson case whereas the second line describe the same for the Klein-Nishina case. $\epsilon(\theta) \equiv m_{e} c^{2} / 2 \gamma(1-\beta \cos \theta)$ denotes the change from Thomson case to Klein-Nishina case, and the quantities

$$
\begin{aligned}
\Delta E^{(1)} & =\frac{\gamma \epsilon^{*}}{1+\epsilon^{*} / m_{e} c^{2}}, \\
\Delta E^{(2)} & =\gamma m_{e} c^{2}\left[1-\frac{2 \epsilon^{*} / m_{e} c^{2}}{\left(1+2 \epsilon^{*} / m_{e} c^{2}\right) \ln \left(1+2 \epsilon^{*} / m_{e} c^{2}\right)}\right]
\end{aligned}
$$


are approximately the average energy loss of an electron in a single Compton collision for $2 \epsilon^{*}<m_{e} c^{2}$ and $2 \epsilon^{*}>m_{e} c^{2}$, respectively. In the above given formulas effects of polarization are not considered and are about a factor of two (e.g. see Kardashev, Mitrofanov, \& Novikov 1984).

With the presence of a magnetic field the electrons may gyrate around the magnetic field lines. In the case when the gyro-frequency equals the Doppler shifted frequency of the infalling photon, i.e. $\epsilon_{\text {res }}=(\hbar e B(r)) /\left(\gamma(1-\beta \cos \theta) m_{e} c\right)$ (with $B(r)$ the magnetic field strength at the location of the electron), the cross section undergoes a resonance. With the use of the Breit-Wigner formula to model this resonance and a substitution of the integration over $d \epsilon$ in equation (1) by a multiplication of the maximum resonant cross section with the resonance width (for details see Supper \& Trümper 1999), we come up with a formula for the energy transfer in the resonant case:

$$
\begin{aligned}
& \frac{d E^{R I C S}}{d r} \cong-\hbar \frac{\sigma_{T}}{\mu} \int_{0}^{4 \pi}(1-\beta \cos \theta) n(\epsilon, \theta) \times \\
& \begin{cases}\Delta E_{\left(\epsilon_{r e s}\right)}^{(1)} d \Omega & : \quad c_{\text {res }} \leq \epsilon(\theta) \\
\frac{3}{4} \frac{m_{e} c^{2}}{2 \epsilon^{*}} \ln \left(1+\frac{2 \epsilon^{*}}{m_{e} c^{2}}\right) \Delta E_{\left(\epsilon_{\text {res }}\right)}^{(2)} d \Omega & : \quad \epsilon_{\text {res }}>\epsilon(\theta)\end{cases}
\end{aligned}
$$

Here, $\mu=\frac{2}{3} \gamma \frac{e^{2}}{m_{e} c^{3}}$ is the relaxation time of the resonance, $\epsilon(\theta)$ is mentioned below equation (1), and $\Delta E_{\left(\epsilon_{r e s}\right)}^{(1 / 2)}$ come out of equations (2) and (3) with $\epsilon^{*} \equiv$ $\gamma(1-\beta \cos \theta) \epsilon_{\text {res }}$.

\section{The thermal photon distribution}

To calculate equations (1) and (4) a thermal photon distribution $n(\epsilon, \theta)$ is required. For a symmetrical case of an electron located centered and above the polar cap of a neutron star in height $d$, the distribution can be given by the expression (see Supper \& Trümper 1999):

$$
n(\epsilon, \theta)=W_{\epsilon}^{\prime} \times g(d, \theta)=\left[\frac{\epsilon^{2}}{(2 \pi \hbar c)^{3}\left(e^{\epsilon / k T}-1\right)}\right] \times\left[2 \pi \frac{\sin \theta}{\cos (\theta+\psi)}\right],
$$

with $W_{\epsilon}^{\prime}$ the spectral term depending on the spectral model (here a black body is used), and $g(d, \theta)$ the geometric term, where $\psi=\arcsin (\sin \theta s / a)$, with $a$ the neutron star radius and $s$ the distance between the electron and the considered emission point at the thermal cap ( $\theta$ is therefore the angle between $d$ and $s$ ). The angle $\theta$ must be always smaller than $\arctan \left(r_{t c} / d\right)$, with $r_{t c}$ the thermal cap radius, and also smaller than the expression $\arcsin (a /(d+a))$ which represents the angle to the horizon.

\section{The differential equation}

Equations (1) and (4) give the differential damping of an electron in a neutron star magnetosphere due to ICS. For the opposite process, the acceleration, we 

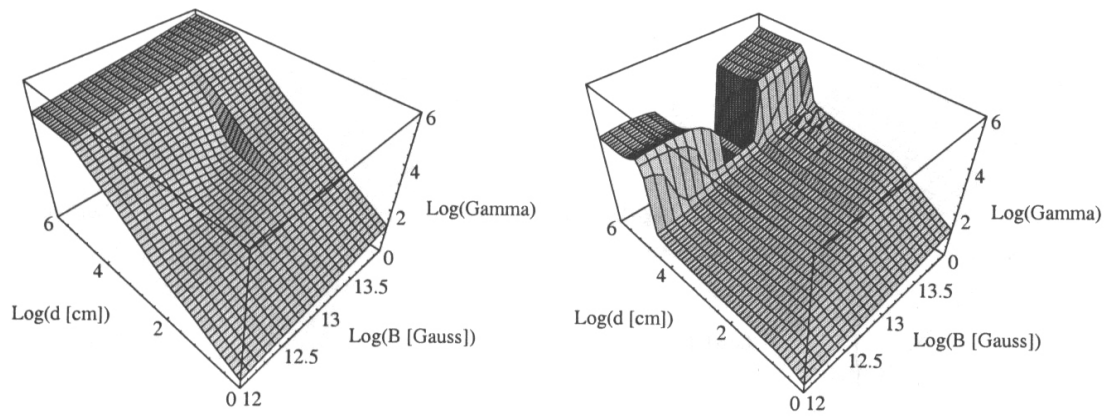

Figure 1. Electron Lorentz factor $\gamma(d, B)$ as a function of height $d$ above the polar cap surface and magnetic field strength $B$ for $k T=$ $100 \mathrm{eV}$ (left) and $k T=300 \mathrm{eV}$ (right). The pulsar rotation period is set to $P=0.150 \mathrm{~s}$ and the radius of the hot thermal cap is set to $10^{5} \mathrm{~cm}$.

use the model of Goldreich \& Julian (1969) and the conditions of Michel (1982). This is a linear acceleration model with an acceleration height $r_{p c}$ and a natural scale length $\lambda$ (within an electron receives kinetic energy in order of its rest energy):

$$
r_{p c}=\left(\frac{\Omega a^{3}}{c}\right)^{1 / 2} \quad, \quad \lambda=\left(\frac{m_{e} c^{3}}{4 e \Omega B}\right)^{1 / 2},
$$

where $\Omega=2 \pi / P$ stands for the angular velocity of the pulsar. With this, the differential equation for the acceleration reads

$$
\frac{d \gamma^{a c c .}}{d r}= \begin{cases}\frac{1}{\lambda} & : \quad r \leq r_{p c} \\ 0 & : \quad r>r_{p c}\end{cases}
$$

Altogether, we end up with the desired differential equation for the energies of accelerated electrons being damped by inverse Compton scattering within an neutron star magnetosphere:

$$
\frac{d \gamma}{d r}=\frac{d \gamma^{a c c}}{d r}+\frac{1}{m_{e} c^{2}}\left(\frac{d E^{I C S}}{d r}+\frac{d E^{R I C S}}{d r}\right) .
$$

\section{Results and conclusions}

Figures 1 and 2 show the computational results of equation (8) for various parameter settings (see figure capture). For all figures the height of the acceleration zone equals the polar cap radius $r_{p c}$ (eq. 6).

Figure 1 shows the developement of the electron energy during acceleration. In the case of a thermal cap temperature $k T=100 \mathrm{eV}$ (left panel) only a slight damping exists within the acceleration zone, which has minor influence on the end energy of the electrons above the zone. This changes dramatically in the 

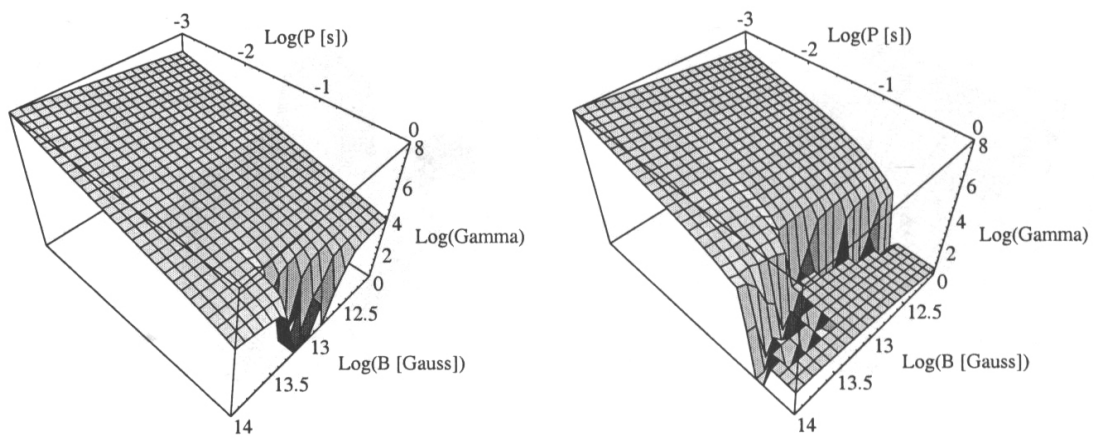

Figure 2. End electron Lorentz factor $\gamma(P, B)$ as a function of pulsar rotation period $P$ and magnetic field strength $B$ for $k T=100 \mathrm{eV}$ (left) and $k T=300 \mathrm{eV}$ (right). The radius of the hot thermal cap is set to $10^{5} \mathrm{~cm}$.

case of $k T=300 \mathrm{eV}$ (right panel), where a strong braking can be found not only within the acceleration zone but also above in the situation where the magnetic field strength is between $10^{12.5}$ and $10^{13.5}$ Gauss. In the case of $k T=1000 \mathrm{eV}$ (not shown in the figure) a total brake down of the electron end energies can be found for $B=10^{12} \ldots 10^{14}$ Gauss. For a more detailed description see Supper \& Trümper (1999).

Figure 2 gives the end energies of the electrons above the acceleration zone as a function of the pulsar rotation period $P$ and the magnetic field strength $B$. For pulsars with $P<0.1$ s no braking can be found (also in the case of $B=10^{8}$ Gauss for milisecond pulsars, not shown in the figure). Above this threshold a (parameter) zone exists where the electrons are braked down totally.

We come up with the following conclusions: Inverse Compton scattering (resonant and non-resonant) is able to substantially lower the end energies of accelerated electrons within a neutron star magnetosphere (using the model of Goldreich \& Julian 1969) in the case of temperatures $k T>100 \mathrm{eV}$ of the thermal cap, rotational periods $P>0.1 \mathrm{~s}$, and for a distinct range of magnetic field strengths. Only for $P<0.1 \mathrm{~s}$ (and therefore for millisecond pulsars) braking due to inverse Compton scattering can be neglected. A more detailed description including energy loss due to curvature radiation is presented in Supper \& Trümper (1999).

\section{References}

Feenberg, E., \& Primakoff, H. 1948, Physical Review 73, No. 5, 449

Goldreich, P., \& Julian W. H. 1969, ApJ, 157, 869

Kardashev, N. S., Mitrofanov, I. G., \& Novikov, I. D. 1984, Astronomische Zeitschrift, 61, 1113

Michel, F. C. 1982, Rev.Mod.Phys., 54, 1

Supper, R., \& Trümper, J. 1999, A\&A, submitted 